
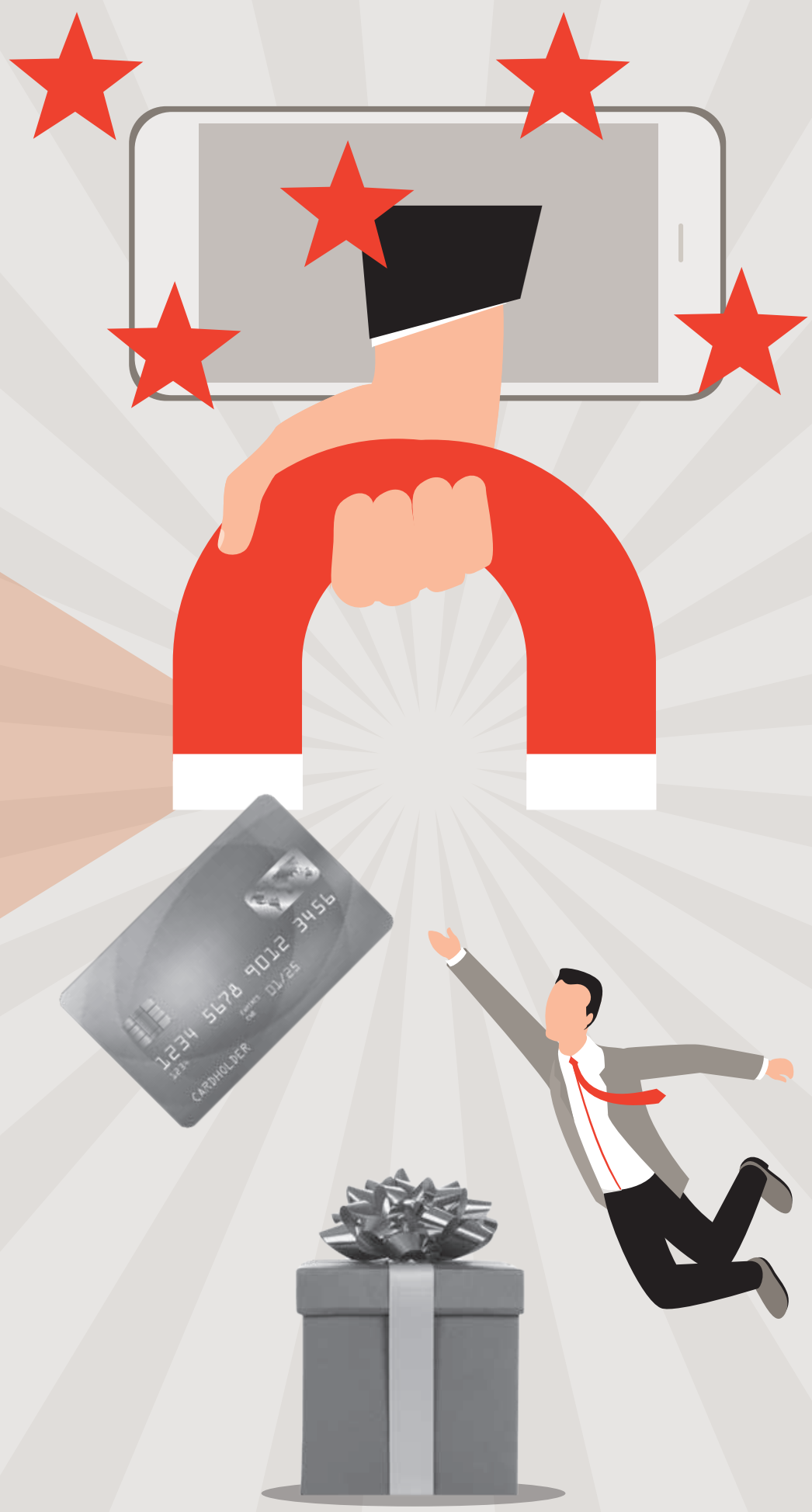


\title{
Keeping Loyalty Programs Fit for the Digital Age
}

\author{
Matilda Dorotic
}

KEYWORDS

Retailing, Loyalty Programs,

Consumer Spending, Rewards, Digitalization

THE AUTHOR

Matilda Dorotic

Professor of Marketing,

Norwegian Business School, Oslo, Norway matilda.dorotic@bi.no
Loyalty programs: Relicts from the past? /// The omnipresence of loyalty programs (LP) across markets shows that LPs have been one of the most prominent business trends of the last two decades. Besides their traditional stronghold among airlines and grocery retailers, loyalty reward schemes have spread among nonprofit organizations like museums, charities and sport clubs, among online and offline services, and even among utility providers and business-to-business markets. On average, two-thirds of Europeans belong to at least one LP. In the UK LP penetration reached $90 \%$ and even $94 \%$ in Finland, according to a worldwide Nielsen study in 2016. The 2017 US census by Colloquy reports 3.8 billion LP memberships, with the strongest penetration in the retailing sector to which more than 1.6 billion memberships belong. Beyond reinforcing customer loyalty and retention, LPs can help a retailer increase its share in a customer's wallet and to cross-sell and up-sell additional products to customers. However, as the number of companies offering LPs soar, the battle for a "place in the consumer wallet" is intensifying, resulting in the fact that more than half of all the memberships that customers sign up for are eventually abandoned, according to Colloquy's reports. This tendency, coupled with increases in investment costs necessary to leverage benefits from LPs, makes some managers question whether supposed gains from LPs are sustainable. These managers wonder whether investments in LPs should rather be replaced with new musthaves such as mobile marketing, gamification and social media leverage.

Beware of bombastic promises, but know that loyalty programs pay off /// Popular press and CRM consulting firms in the last two decades have boasted about large differences in purchase levels and profitability between LP 
$\{$ Box 1$\}$

\section{CALCULATE THE EFFECTIVENESS OF YOUR LP CORRECTLY}

To calculate the true return on your LP investment, it is not sufficient to look at the average differences in spending between members and nonmembers. Rather, start by observing individual customer's purchase behavior before they joined the LP. Identify members and nonmembers who in the period before joining the LP had similar purchase levels, demographics and other information that you can get. If you pair the LP member with such a similar nonmember and compare how member's purchase behavior changed relative to the nonmember's in the periods after joining the LP, you will be able to gauge the true effect more realistically.

FIGURE 1:

\section{Compare purchase behavior of similar members versus nonmembers before and after joining an LP program to get the true effects}

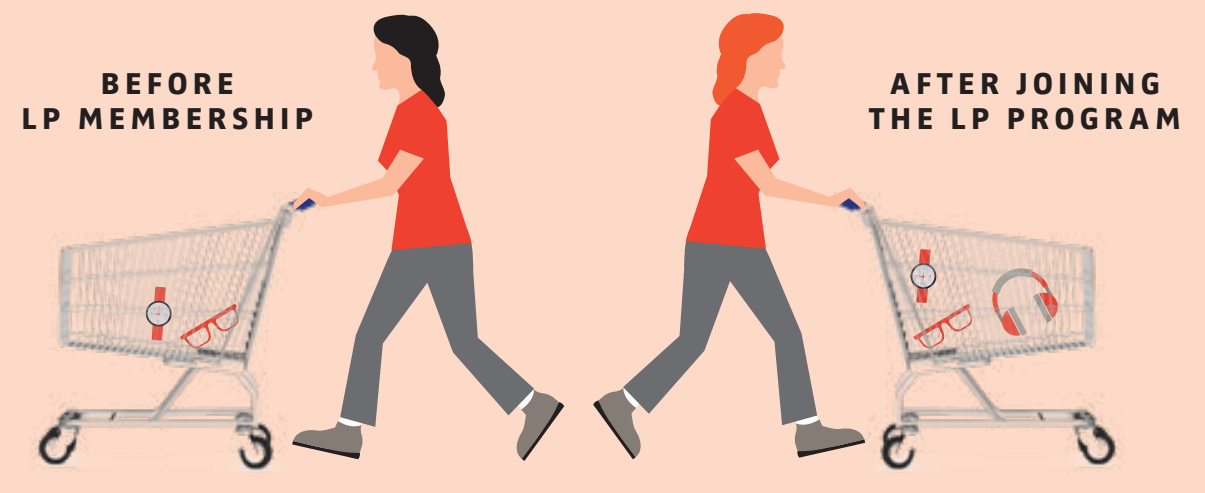

members and nonmembers, attributing the difference in purchase levels to the LP. The large difference in spending levels, between LP members and nonmembers, comes primarily from the fact that loyal, high-spending customers are more likely to become LP members. The true impact on performance that could be attributed to LPs is much smaller, although it is significant and positive. This impact comes from $L P$ 's ability to increase spending and frequency of purchasing of LP members after they join the LP. In general, research evidence shows that retailers of FMCGs can expect from 6-25\% increase in sales and airlines around $4 \%$. Specifically, research among Dutch supermarket chains showed that loyalty program membership on average increases share-of-wallet by $4 \%$. This effect is seven times smaller than the increase in sales that would be attributed to the LP if the underlying dif- ference between members and nonmembers were not taken into account.

> LPs are primarily attracting existing customers /// One misconception to avoid is the belief that an LP is able to attract significant numbers of new customers who are otherwise less likely to shop with the retailer. LPs predominantly engage existing customers - those who feel they do not have to significantly change their standard purchase behavior to earn rewards. Customers most likely to join retailers' LPS are those who already purchase, those who live close by and those for whom buying at the retailer is convenient anyway. In any case, retailers should expect the greatest increase in purchase frequency and volume within a few months of the LP introduction. 
$>$ The LP effect is stronger for medium and light buyers /// Moreover, managers may be surprised to realize that the largest increases in purchase behavior may not come from their "best" customers and high spenders. LP members most likely to significantly increase their spending and share of wallet are customer segments that are often underestimated and perceived as less relevant: medium and light buyers. The explanation is straightforward: Heavy buyers and high spenders already devote a large part of their overall consumption in a category to their favorite company, so they have less room to grow their expenditures. Light and medium buyers may, in contrast, increase their share of purchases by switching from competitors or increasing their consumption in the category.

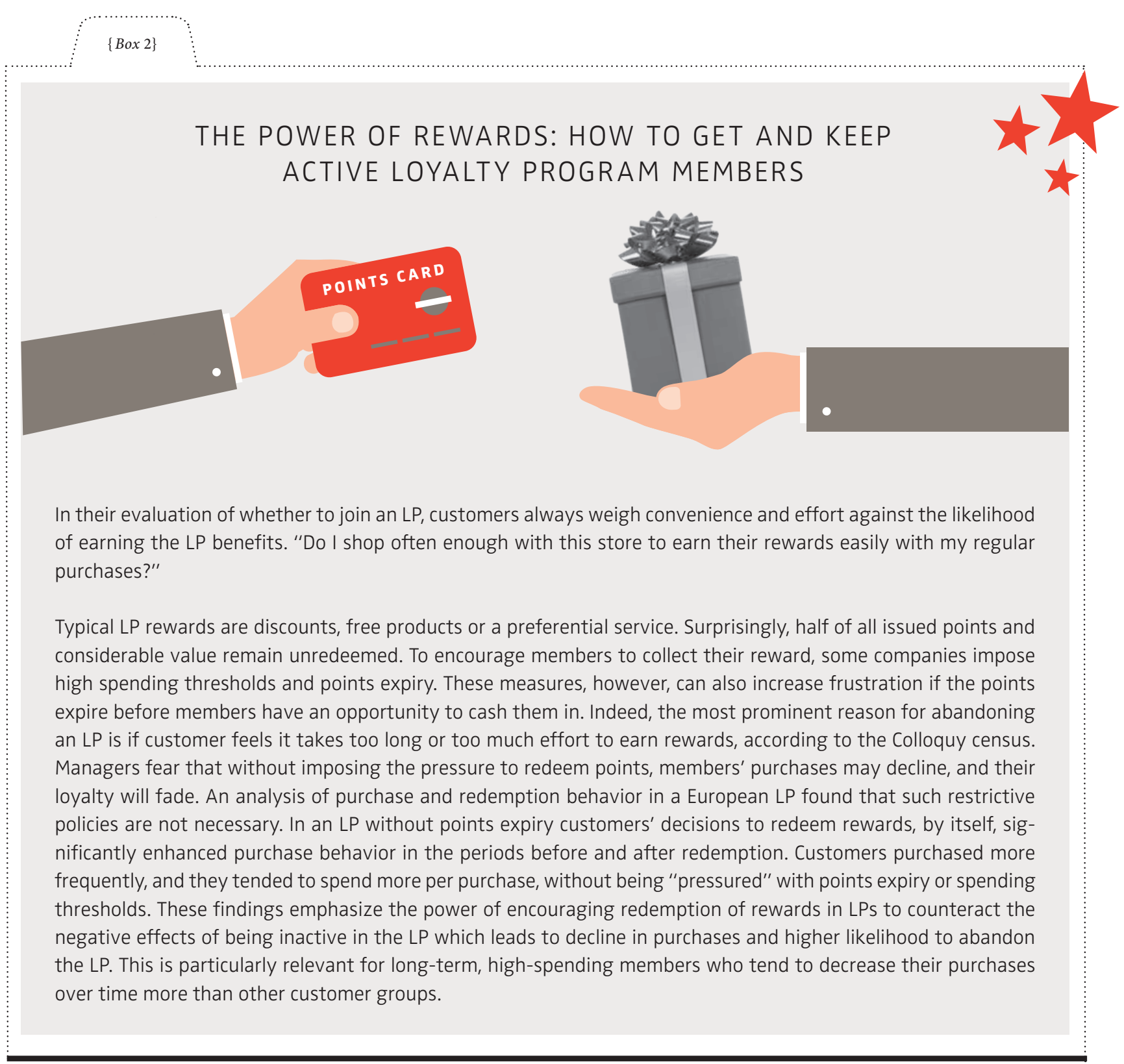




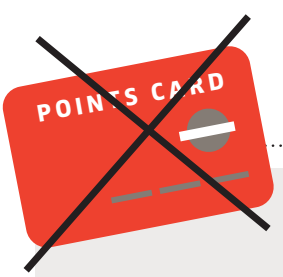

FIGURE 2:

\section{Future loyalty program success factors}

\section{GO DIGITAL}

\section{INSTEAD OF CARDS}

$\rightarrow$ integrate with mobile applications

$\rightarrow$ integrate with digital payment systems

$\rightarrow$ Integrate with real-time in-store offers

\section{BUILD NETWORKS}

$\rightarrow$ Include complementary partners to increase value

\section{INTEGRATE MONETARY AND NONMONETARY AWARDS}
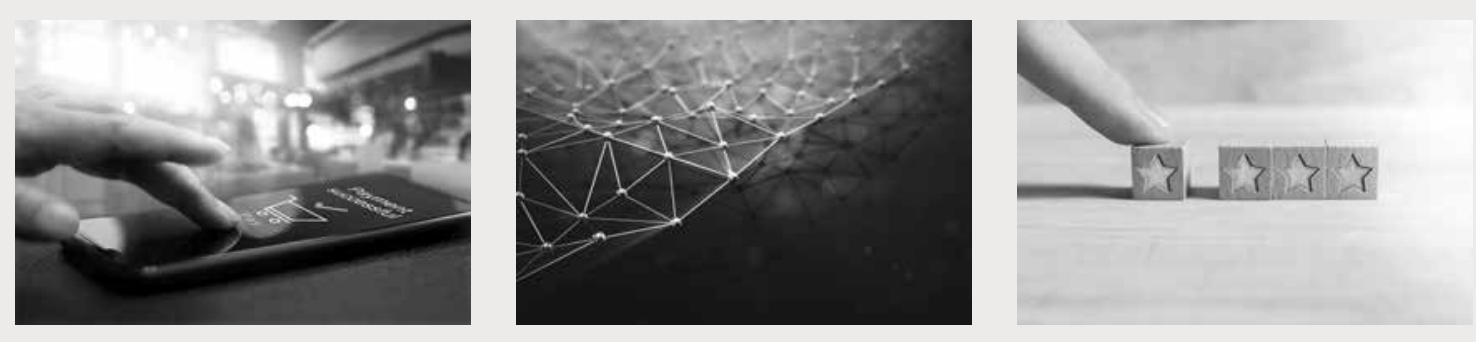

The future of loyalty programs: How to keep LPs worthwhile in the digital age /// The core idea of leveraging information gained through loyalty initiatives remains stronger than ever. As LP membership penetration reaches all-time highs, customers demand more value from LPs. There are several ways in which LPs need to increase value to remain attractive in a more and more digital and highly competitive retailing space.

> Think beyond plastic cards /// What sceptics about the future of LPs may rightfully highlight is that the effectiveness of classic LPs as plastic cards is declining. The future of LPs is linked to leveraging opportunities that mobile devices offer in increasing the convenience and communication with LP members. Digital transformation for LPS seems particularly important, because as many as around one third of LP members are willing to abandon an LP if it does not offer a mobile-friendly solution. In the future, the integration of LPs with digital payment systems like mobile wallets seems particularly promising. Such integrations would allow customers to seamlessly earn and redeem rewards when they are using a mobile wallet. The savvy LP retailer will go one step further and integrate their program and digital payment systems with realtime targeting of offers in the store. Flexible redemption schemes would offer customers an opportunity to buy in the store with a combination of points and money and encourage redemption of rewards in real time. Starbucks and the UK supermarket chain Tesco, for example, have already implemented NFC technology successfully to integrate contactless payments with mobile wallet and their LPs' earning and redeeming options.

$>$ The power of networking: Increase the value through LP partnerships /// Another way to become more attractive is by forming partnerships with other companies at which customers can collect and/or redeem points. Lufthansa's Miles and More, for instance, currently has around 300 partners, of which 270 are nonaviation partners. They regard their LP as a platform for many partners, offering a broad range of customer loyalty possibilities. Similarly, the Payback coalition of partners has reached 30 million active 
$\gg$

\section{The power of networking is increasing the value of an LP, both for customers and participating companies.}

$<$

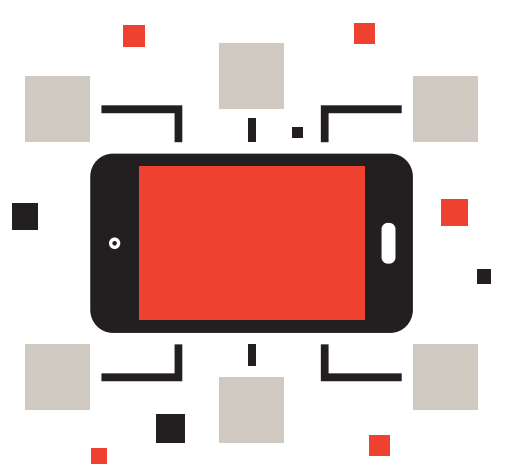

members in Germany and distributed 50 billion coupons in 2017. Such partnership LPs hold appeal for retailers, particularly if potential partners might benefit from synergies. An initial study of an LP coalition of an airline and complementary partners shows that customer usage and satisfaction with the airline as the core service increased cross buying from complementary partners like hotels, which in turn reinforced usage of the core service. Moreover, partnerships seemed to some extent be able to shield partners from the negative effects of service failures. The power of networking is increasing the value of an LP, both for customers and participating companies, and the future will likely see more networking in LPs.

$>$ Be more creative in what to reward /// In the future of linked data, we also expect to see higher flexibility in terms of the types of customer engagements that will be rewarded. Many companies already move towards rewarding customer engagement not only on the base of past transactions, but also for their activities on social media. They accredit, for example, points for WOM referrals, or for writing social media posts or customer reviews, and they acknowledge other customer activities that are not directly related to purchases, such as health-promoting activities like running.
Customers still enjoy some form of loyalty rewarding and expect retailers to acknowledge their purchases as investments in relationships. Particularly for retailers who rely on high quality and customer experience building through a differentiation strategy, some form of customer relationship building is unavoidable. If we see the LPs for the gist that they could offer - enhancing customer experiences and building loyalty - they will remain as attractive and essential as when they were first introduced, although we may have considerably fewer plastic cards in our wallets.

I.

\section{FURTHER READINGS}

Dorotic, M., Bijmolt, T. H. and Verhoef, P. C. (2012):

"Loyalty Programmes: Current Knowledge and Research Directions", International Journal of Management Reviews, Vol. 14, 217-237.

Dorotic, M., Verhoef, P. C., Fok, D. \& Bijmolt, T. H. A. (2014):

"Reward redemption effects in a loyalty program when customers choose how much and when to redeem", International Journal of Research in Marketing, Vol. 31 (4), 339-355.

Leenheer, J., Van Heerde, H. J., Bijmolt, T. H., \& Smidts, A. (2007):

"Do loyalty programs really

enhance behavioral loyalty? An empirical analysis accounting for self-selecting members"; International

Journal of Research in Marketing, Vol. 24 (1), 31-47.

https://www.colloquy.com/

latest-news/u-s-customer-loyalty-program-memberships-reachdouble-digit-growth-at-

3-8-billion-2017-colloquy-loyalty-census-reports/ 